\title{
Expanding the Dimensions of a High Dynamic Range Detector with a Limited Number of Pixels via Scripting
}

\author{
Xi Chen ${ }^{1}$, Tim B. Eldred ${ }^{1}$, Mostafa Abdelhamid ${ }^{2}$ and James M. LeBeau ${ }^{1}$ \\ 1. Department of Materials Science and Engineering, North Carolina State University, Raleigh, NC, \\ USA \\ 2. Department of Electrical and Computer Engineering, North Carolina State University, Raleigh, NC, \\ USA
}

Hybrid 4D STEM detectors have recently enabled E/M field mapping [1], atomic resolution ptychography [2], strain mapping [1], and fully quantitative imaging [3]. In particular, the Electron Microscopy Pixel Array Detector (EMPAD) achieves a dynamic range of 1,000,000:1 within a single frame, allowing the direct electron beam to be imaged while still maintaining single electron sensitivity [4]. Sample drift distortions are minimized by the high frame rate, which enables capturing scattered electrons at each scanning position in STEM image [2-5]. While detector technologies are capable of single electron sensitivity while counting at $>1,000 \mathrm{fps}$, the number of pixels is fairly limited $(128 \mathrm{x}$ 128).

In this presentation, we will demonstrate how a detector with a small number of pixels can be used to capture the entire electron scatter distribution at any camera length using scripting. We will discuss how we have built a custom python script to control the microscope to shift the diffraction pattern while the EMPAD acquires each dataset, Figure 1(a). We will also examine the challenges associated with reconstructing the datasets, including rotating the pattern shift axes and lens hysteresis. By including some overlap between the patterns, we will highlight our automated routine to accurately and precisely align the data post-acquisition. This process allows for the detection range to be limited only by the objective lens bore even with this small detector. As shown in Figure 1(b), a significant portion of reciprocal space is shown at high k-space sampling rate, with the final, assembled diffraction pattern retaining the fine details as in Figure 1(c).

We will also discuss how to use the technique to capture only certain segments of reciprocal space to save time and storage. For example, to capture only high angle diffuse scattering, it is possible to acquire along an annulus of the diffracted intensity as in Figure 2(a). In this case, the diffraction pattern was shifted in a ring pattern to avoid the central beam. Finally, we will also discuss how this method can combine full 4D diffraction datasets to capture a larger diffraction pattern at each STEM image position. As shown in Figure 2(b)-(c), the CBED diffraction pattern was captured to beyond the FOLZ with good reciprocal space sampling using only a 128x128 pixel detector. The resulting patterns are each 32-bit $325 \times 325$ floating point pixels yielding a final image data size of $10011 \mathrm{gb}$. This enables simultaneous HAADF STEM and high precision strain mapping using the same dataset [6].

\section{References:}

[1] H Ryll et al., JINST 11 (2016), P04006

[2] Y Jiang et al., Nature 559 (2018), P343-349

[3] Z Chen et al, Ultramicroscopy 169 (2016), P107-121 
[4] MW Tate et al., Microsc. Microanal 22 (2016), P237

[5] H Yang et al, J. Phys.: Conf. Ser. 644 (2015), P012032

[6] We acknowledge support for this work from the Air Force Office of Scientific Research (FA955017-1-0225. This work was performed in part at the Analytical Instrumentation Facility (AIF) at North Carolina State University, which is supported by the State of North Carolina and the National Science Foundation (award number ECCS-1542015). The AIF is a member of the North Carolina Research Triangle Nanotechnology Network (RTNN), a site in the National Nanotechnology Coordinated Infrastructure (NNCI).

$128 \times 128$ pixels, 25 shifts

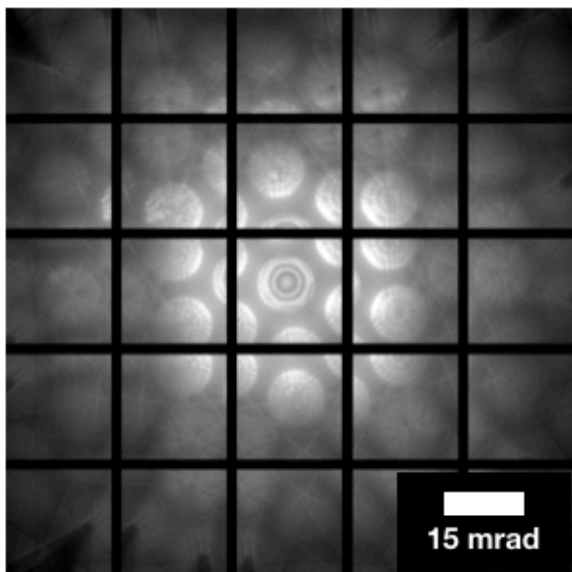

(a)

\section{$458 \times 458$ pixels}

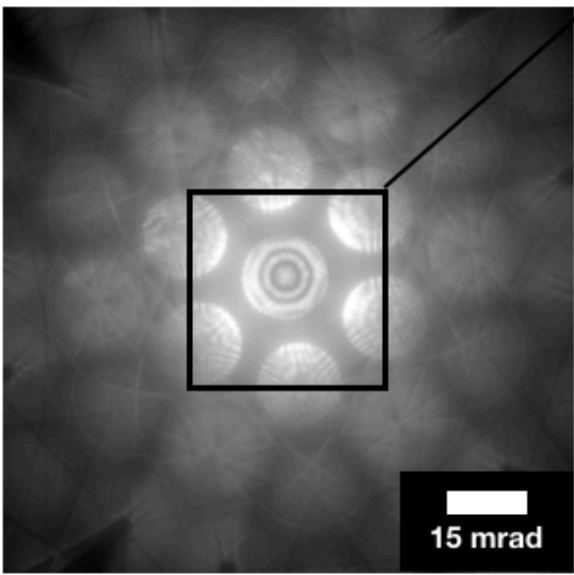

(b)

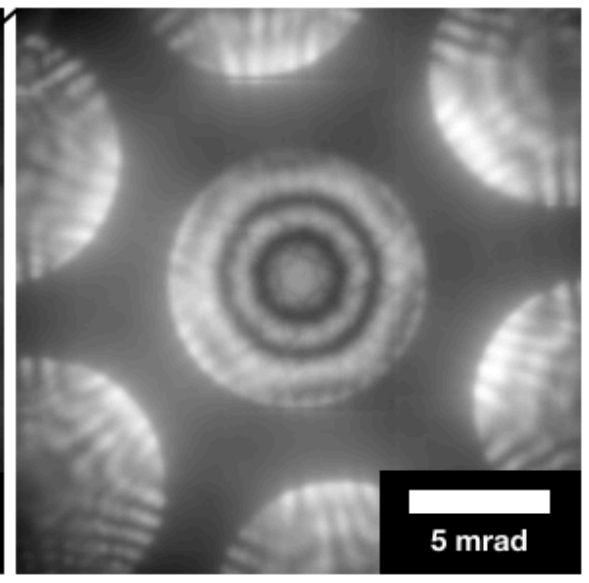

(c)

Figure 1. (a) Individual EMPAD diffraction patterns for $<111>$ silicon (b) The resulting merged diffraction pattern after accurate and precise pattern alignment, (c) Details within the central disk are readily resolved with and outer limit to $\mathbf{g}_{264}$.

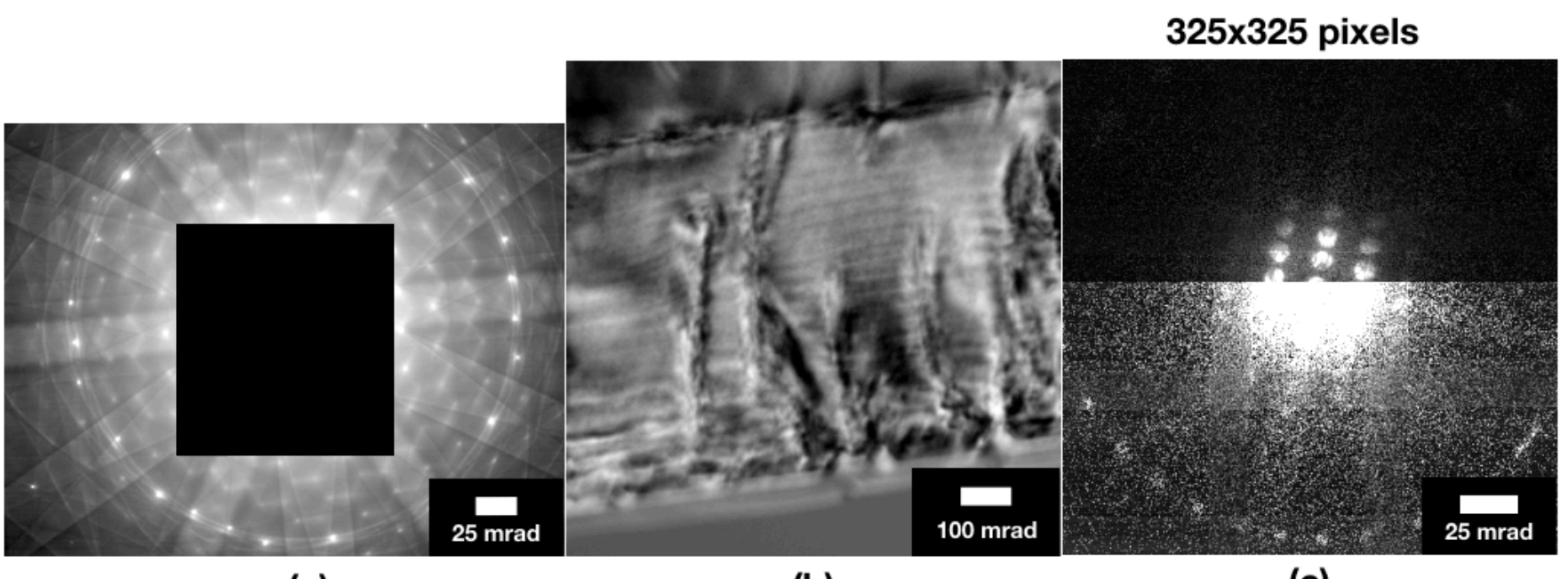

(a)

(b)

(c)

Figure 2. (a) A single diffraction pattern stitched together from 9 individual pattern shifts (b) Annular EMPAD diffraction pattern (c) Center of mass image of an InGaN/GaN superlattice and 